\title{
Geomorphological mapping based on DEMs and GIS: A review
}

\author{
Takashi Oguchi ${ }^{\mathrm{a}, *}$ \\ ${ }^{a}$ Center for Spatial Information Science, The University of Tokyo, Japan.oguchi@csis.u-tokyo.ac.jp \\ * Corresponding author
}

Keywords: Geomorphology, mapping, GIS

\begin{abstract}
:
Geomorphology is a scientific discipline dealing with the characteristics, origin, and evolution of landforms. It utilizes topographic data such as spot height information, contour lines on topographic maps, and DEMs (Digital Elevation Models). Topographic data were traditionally obtained by ground surveying, but introduction of aerial photogrammetry in the early 20th century enabled more efficient data acquisition based on remote sensing. In recent years, active remote sensing methods including airborne and terrestrial laser scanning and applications of satellite radar have also been employed, and aerial photogrammetry has become easier and popular thanks to drones and a new photogrammetric method, SfM (Structure from Motion). The resultant topographic data especially raster DEMs are combined with GIS (Geographic Information Systems) to obtain derivatives such as slope and aspect as well as to conduct efficient geomorphological mapping. Resultant maps can depict various topographic characteristics based on surface height and DEM derivatives, and applications of advanced algorithms and some heuristic reasoning permit semi-automated landform classification. This quantitative approach differs from traditional and more qualitative methods to produce landform classification maps using visual interpretation of analogue aerial photographs and topographic maps as well as field observations.

For scientific purposes, landforms need to be classified based on not only shape characteristics but also formation processes and ages. Among them, DEMs only represent shape characteristics, and understanding formation processes and ages usually require other data such as properties of surficial deposits observed in the field. However, numerous geomorphological studies indicate relationships between shapes and forming-processes of landforms, and even ages of landforms affect shapes such as a wider distribution of dissected elements within older landforms. Recent introduction of artificial intelligence in geomorphology including machine learning and deep learning may permit us to better understand the relationships of shapes with processes and ages. Establishing such relationships, however, is still highly challenging, and at this moment most geomorphologists think landform classification maps based on the traditional methods are more usable than those from the DEM-based methods. Nevertheless, researchers of some other fields such as civil engineering more appreciate the DEM-based methods because they can be conducted without deep geomorphological knowledge. Therefore, the methods should be developed for interdisciplinary understanding. This paper reviews and discusses such complex situations of geomorphological mapping today in relation to historical development of methodology.
\end{abstract}

